\title{
Violencia de pareja e interposición de denuncias en mujeres víctimas en Nicaragua ${ }^{9}$
}

\author{
Esther Rivas Rivero \\ Doctora en Psicología \\ Universidad de Alcalá, España \\ Correo electrónico: esther.rivas@uah.es \\ Enrique Bonilla Algovia \\ Magíster en Psicopedagogía \\ Universidad de Alcalá, España \\ Correo electrónico: enrique.bonilla@uah.es \\ Jesús Redondo Pacheco \\ Doctor en Psicología \\ Universidad Pontificia Bolivariana \\ Correo electrónico: jesus.redondo@upb.edu.co
}

Sonia Panadero Herrero

Doctora en Psicología

Universidad Complutense de Madrid, España

Correo electrónico: spanadero@edu.ucm.es

\section{José Juan Vázquez Cabrera}

Doctor en Psicología

Universidad de Alcalá, España

Correo electrónico: jj.vazquez@uah.es

Recibido: 11/03/2019

Evaluado: 01/04/2019

Aceptado: 17/05/2019

\section{Resumen}

La violencia contra las mujeres en la pareja es un problema de salud pública a nivel mundial, aunque en algunas culturas, su frecuencia impide su visibilización y que se emprendan medidas para erradicarla. El objetivo del trabajo pretende analizar las circunstancias y tipos de maltrato en una muestra de 136 víctimas de violencia en la pareja, con una media de edad de 31.7 años (DT = 8.921), que habitan en las áreas marginales de León (Nicaragua), así como estudiar la relación entre dicha violencia y la interposición de denuncias por la violencia ejercida a éstas. Los resultados evidencian que la interposición de denuncias correlaciona con la frecuencia del maltrato. En este sentido, las víctimas parecen identificar la violencia y adoptar medidas cuando ésta se produce de manera reiterada. Identificar el maltrato es un objetivo prioritario en culturas donde las agresiones se encuentran normalizadas en las dinámicas de pareja como consecuencia de la socialización.

Palabras clave

Violencia contra las mujeres en la pareja, interposición de denuncias, maltrato, pobreza, Nicaragua.

9 Para citar este artículo: Rivas, E., Bonilla, E., Redondo, J., Panadero, S. \& Vázquez, J. J. (2020). Violencia de pareja e interposición de denuncias en mujeres víctimas en Nicaragua. Informes Psicológicos, 20(1), pp. 131-146 http://dx.doi. org/10.18566/infpsic.v20n1a09 


\title{
Partner violence and filing complaints in women victims in Nicaragua
}

\begin{abstract}
Violence against women in the couple is a worldwide public health problem, although in some cultures, its frequency prevents its visibility and that measures are taken to eradicate it. The objective of the work is to analyze the circumstances and types of abuse in a sample of 136 victims of violence in the couple, with an average age of 31.7 years $(S D=8.921$ ), who live in the marginal areas of León (Nicaragua). The research also seeks to study the relationship between such violence and the filing of complaints for the violence exerted on them. The results show that the filing of complaints correlates with the frequency of abuse. In this sense, the victims seem to identify the violence and take measures when it occurs repeatedly. Identifying abuse is a priority objective in cultures where aggressions are normalized in couple dynamics as a consequence of socialization.
\end{abstract}

Keywords

Violence against women in the couple, filing complaints, abuse, poverty, Nicaragua.

\section{Violência no casal e registro de queixas em mulheres vitimas na Nicarágua}

\section{Resumo}

A violência contra as mulheres no casal é uma problemática de saúde pública de nível mundial, embora que em algumas culturas sua frequência impede sua visualização e sejam executadas medidas para sua erradicação. Este trabalho tem 0 intuito de analisar as circunstâncias e tipos de abuso em uma amostra de 136 vítimas de violência no casal, com uma média de idade ao redor dos 31.7 anos (DT = 8.921), que moram nas áreas marginais de León (Nicarágua), também se tem o objetivo de estudar a relação entre tal violência e 0 registro de queixas por violência exercida contra elas. Os resultados evidenciam que o registro de queixas tem correlação com a frequência do abuso. Nesse sentido, as vítimas parecem identificar a violência e tomar medidas quando ela ocorre repetidamente. Identificar abuso é um objetivo prioritário em culturas em que as agressões estão normalizadas na dinâmica de casais como consequência da socialização. 


\section{ntroducción}

A lo largo de la historia ha prevalecido un modelo patriarcal donde la autoridad depende de los hombres, ya que el patriarcado determina un conjunto de prácticas cotidianas que niegan los derechos de las mujeres con el fin de generar un desequilibrio entre ambos sexos (Medrano, 2012). El patriarcado está enraizado en las sociedades y ejerce una fuerte coacción estructural que afecta a la violencia ejercida contra las mujeres (De Miguel, 2005). Por lo tanto, el patriarcado, como estructura social de dominación masculina y subordinación femenina, es el marco explicativo de la desigualdad de las estructuras macrosociales (Ferrer, 2017) que sostiene la violencia de género.

Según Galtung (1990), existen distintas formas de ejercer la violencia relacionadas entre sí: (1) la violencia directa, referida a la violencia observable contra las mujeres, (2) violencia estructural, que se refleja en situaciones de discriminación en culturas basadas en el patriarcado, en las que la mujer queda relegada al ámbito privado y (3) violencia cultural, que engloba las anteriores formas de violencia y justifica la discriminación ejercida contra estas. La violencia directa es la forma más explícita de someter a las mujeres a través de distintos tipos de maltrato. Específicamente, la abundante literatura científica señala tres tipos de violencia ejercida contra las mujeres: la violencia física, que implica todo tipo de conductas intencionales que produzcan daño corporal o lesión a través de golpes de diversa intensidad; la violencia psicológica, que hace referencia a conductas como insultar, humillar, realizar críticas constantes, gritos, chantajes, amenazas, etc.; y la violencia sexual, que consiste en conductas que abarcan desde la imposición de relaciones sexuales, hasta el abuso y la violación por medio de la fuerza física (Escobar, Quinteros, Sánchez \& Tardón, 2011; Eyre et al., 2013; Shorey, Brasfield, Zucosky, Febres \& Stuart, 2015; Walker, 2012).

Según Domínguez, García y Cuberos (2008), el tipo de violencia más habitual es la psicológica (52\%), seguida de la física (18\%). Cuando las mujeres son víctimas de un único tipo de violencia, la más frecuente es la psicológica (Fontanil et al., 2002). Independientemente del tipo de violencia, cuando esta se instaura en la dinámica de la pareja, se entremezcla con episodios de arrepentimiento y ternura que conducirían a la víctima a una situación de dependencia emocional (Saltijeral, Ramos \& Caballero, 1998).

Esta situación desarrolla un estado de confusión en las mujeres al no explicarse el motivo por el que son víctimas de la violencia ejercida por sus parejas (Miramontes \& Mañas, 2018), llegando a culparse por no tratar adecuadamente a su pareja. Aun manifestándose la violencia de manera explícita, las víctimas pueden llegar a soportar la convivencia con el agresor durante años con la esperanza de que su pareja cambie (Echeburúa, Amor \& Corral, 2002). Cuando asumen que la violencia continúa y que aumenta en intensidad y frecuencia, la perciben como incontrolable. Según Labrador, Fernández-Velasco y Rincón (2010), cuando se produce violencia perpetrada por la pareja, tiende a ser repetitiva, con una media de seis incidentes al año.

Uno de los logros del feminismo contemporáneo ha sido impulsar la creación de recursos para combatir la violencia 
contra las mujeres, no como un problema aislado, sino como un problema social y de salud pública. El feminismo ha promovido las denuncias por violencia contra las mujeres dejando al margen su consideración de asunto privado (Souza et al., 2014), ya que acotarla al ámbito privado ha supuesto su invisibilización y su consideración como delito oculto (Bosch, Ferrer, Alzamora \& Navarro, 2005).

Por otro lado, el interés respecto al comportamiento de búsqueda de ayuda por parte de las víctimas que padecen o han padecido violencia es reciente, pero en muchos países, la mayoría oculta su situación (Leonardsson \& San Sebastián, 2017). Según la Organización Mundial de la Salud (OMS, 2005), entre el 55\% y el 95\% de las mujeres que han padecido violencia perpetrada por la pareja no buscan ayuda en las instituciones formales (García-Moreno, Jansen, Ellsberg, Heise \& Watts, 2005). Este periodo, denominado "ruta crítica" (Sagot \& Carcedo, 2000), implica romper el silencio y revelar su situación. No obstante, denunciar está asociado a determinadas situaciones para las cuales las mujeres han de estar preparadas (Blay, 2013). Incluso las víctimas que se han recuperado tras haber sufrido violencia perpetrada por sus parejas o exparejas tienen periodos que se encaminan a salvar la relación y dar nuevas oportunidades (Cala, 2012). Además, muchas mujeres desechan la idea de denunciar porque supone la confrontación con el agresor, la ruptura con la relación y el inicio de un proceso judicial (Blay, 2013). No obstante, ni las características de las mujeres ni de los agresores pueden predecir la conducta en la interposición de denuncias (Sleath \& Smith, 2017). A lo anterior hay que añadir que, en países con bajos ingresos y gran desigualdad de género, pocas mujeres buscan protección (Leonardsson \& San Sebastián, 2017).

En Latinoamérica, según la OMS/OPS (2012), los comportamientos de búsqueda de ayuda por parte de las mujeres que sufren violencia varían significativamente de un país a otro. Por ejemplo, entre las mujeres que habían sufrido violencia en el seno de la pareja en Honduras, el 29.3\% había informado de su situación, mientras que, en El Salvador, el porcentaje fue del 65.5\%. Particularmente, Nicaragua está sujeta a un contexto de desigualdad donde las mujeres asumen la mayor carga de pobreza y están sometidas a diferentes formas de violencia como consecuencia de una sociedad patriarcal (Medrano, 2012). Según el Instituto Nacional de Información y Desarrollo (INIDE, 2015), en los datos registrados en el año 2011, en Nicaragua hubo un total de 30.746 víctimas de violencia de género. La Encuesta Nacional de Demografía y Salud (ENDESA, 2010) ha reportado que el tipo de violencia que mayor peso tiene entre las mujeres nicaragüenses es la violencia psicológica (48\%), seguida de la violencia física (27\%). Según algunos autores, la no interposición de denuncias, la retirada de las mismas o la retractación son un problema importante entre las mujeres víctimas de violencia de género en este país (Vázquez, Rivas, Suárez \& Panadero, 2018). Además, las mujeres mencionan muchas razones por las cuales no acuden a las instituciones, entre ellas, vergüenza, temor a represalias, no saber dónde ir y/o no creer que les ayudarán (Bolaños et al., 2009).

Determinados valores respecto a mantener a la familia unida podrían estar influyendo de manera directa en la decisión de no romper con la relación, principalmente 
cuando las víctimas cuentan con apoyo social, lo cual refleja la normalización de la violencia en muchos hogares en Nicaragua (Rivas, Panadero, Bonilla, Vásquez \& Vázquez, 2018). Por ello, un cambio en la percepción de las mujeres nicaragüenses respecto a las circunstancias de violencia y la búsqueda de ayuda podría facilitar el fin del abuso con el apoyo y fortalecimiento de las instituciones públicas (Salazar, Högberg, Valladares \& Ohman, 2012).

Hay que añadir que los índices de pobreza de la región, que afecta a la mitad de la población, podrían aumentar la vulnerabilidad de las mujeres que han padecido violencia de pareja, quienes encuentran dificultades para abandonar la relación y están expuestas a situaciones de alto riesgo (McNaughton, Billings, Paredes-Gaitan \& Padilla-Zuniga, 2012). En opinión de Sagot (2000), la impunidad social respecto a la violencia contra las mujeres ha mantenido su aceptación en Centroamérica, de modo que la sensación de inseguridad parece conducirlas a desconfiar del sistema y de la justicia. Aunque todos estos países forman parte de los acuerdos por los Derechos Humanos que obligan a los Estados a garantizar el acceso a medidas de atención integral a las víctimas (McNaughton et al., 2012), los recortes presupuestarios y la eliminación de las Comisarías de la Mujer, institución encargada de su atención integral, hacen difícil la eliminación de este problema en Nicaragua. Por ello, los objetivos del presente estudio son conocer la tipología, frecuencia e intensidad de la violencia de pareja padecida por una muestra de mujeres nicaragüenses, conocer las circunstancias de la violencia asociadas a los procesos de interposición de denuncias y predecir las variables que influyen en dicha interposición.

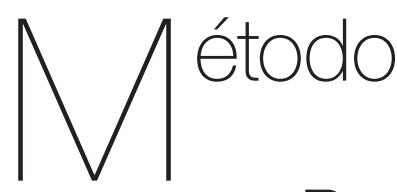

\section{Participantes}

En la investigación participaron 136 mujeres en situación de pobreza, víctimas de la violencia ejercida por sus parejas o exparejas, con edades comprendidas entre los 18 y los 57 años de León (Nicaragua). Los criterios de inclusión muestral fueron ser residentes en la ciudad de León y ser o haber sido maltratadas por el cónyuge o la pareja. Al ser el contexto de pobreza objeto de interés para el presente estudio, el nivel económico de las mujeres fue considerado como criterio de inclusión muestral, determinado por la zona geográfica de la vivienda en la que residían (zonas periféricas y marginales de la ciudad).

Tabla 1.

Características de la muestra

\begin{tabular}{lccc}
\hline & $\mathrm{n}$ & $\%$ & Media (DT) \\
\hline Edad Media (DT) (años) & & & $31.7(8.9)$ \\
Número de hijos (DT) & & & $2.2(1.7)$ \\
ESTADO CIVIL & 33 & $24.3 \%$ & \\
Soltera & 31 & $22.8 \%$ & \\
Casada & 46 & $33.8 \%$ & \\
Unión de hecho & 22 & $16.2 \%$ & \\
Separada & 4 & $2.9 \%$ & \\
Divorciada & & & \\
NIVEL EDUCATIV0 & 4 & $2.9 \%$ \\
Sin estudios & 93 & $68.4 \%$ \\
$\quad$ Educación básica & 24 & $17.7 \%$ \\
Educación media & 15 & $11.1 \%$ \\
Educación superior & & \\
\hline
\end{tabular}


Las participantes tenían una edad media de 31.7 años (DT = 8.921) (Tabla 1). Más de la mitad estaba casada o en unión de hecho estable. El nivel de estudios que predomina entre las entrevistadas fue Educación Básica. Respecto a las circunstancias del maltrato, todas padecieron alguna forma de violencia psicológica y física, y dos de cada tres sufrieron violencia sexual por parte de sus parejas o exparejas (66.9\%). En cuanto a la frecuencia del maltrato, una de cada cuatro lo padecía diariamente (24.2\%), en torno a la mitad lo sufría entre dos y tres veces a la semana (44.7\%), cada quince días el $22.7 \%$ y se producía una vez al mes en el $8.3 \%$ de las participantes. Las mujeres de la muestra comenzaron a vivir con el agresor a una edad media de 19.91 (DT = 4.929), llevaban conviviendo con el agresor 9 años $(M=9.16$; DT = 6.789) y la situación de violencia se prolongó durante más de 6 años $(M=6.25$; $D T=5.481)$. Finalmente, el $34.6 \%(n=47)$ no interpuso denuncia por la violencia padecida, el 42.6\% $(n=58)$ retiró la denuncia una vez interpuesta (retractación) y el $22.8 \%$ denunciaron $(n=31)$ y mantenían la denuncia en curso en el momento de realización de la entrevista.

\section{Procedimiento}

En la investigación se utilizaron diferentes estrategias. En primer lugar, con el apoyo de la Comisaría de la Mujer, se enviaron solicitudes para participar en el estudio a mujeres que en algún momento hubiesen acudido a la Comisaría por la violencia ejercida por sus parejas o exparejas. Por otro lado, la Comisaría de la Mujer facilitó el nombre de las asociaciones que formaban parte de la Red de Apoyo en la eliminación de la violencia contra las mujeres. Se realizó una visita a dichas asociaciones y se informó del objetivo del estudio. Tras la participación de las asociaciones, se solicitó a las profesionales el contacto con mujeres víctimas de violencia de pareja que hubieran sido atendidas en sus dependencias para participar voluntariamente en el estudio. También se contactó con otras mujeres que se encontraban en la misma situación a través de quienes estaban formando parte de la muestra. Las mujeres entrevistadas fueron informadas del carácter confidencial y voluntario de la entrevista y se solicitó su consentimiento informado. La duración de estas osciló entre 45 y 80 minutos.

\section{Instrumentos}

En la recogida de información se empleó una entrevista estructurada que permitió subsanar los posibles problemas derivados de las dificultades de lectura y/o comprensión. Dicha entrevista comprendía los siguientes apartados:

Datos sociodemográficos: las principales variables que se tuvieron en cuenta fueron la edad, el número de hijos, el estado civil y nivel de estudios de las participantes.

Historia y circunstancias de la violencia: se incorporó a la entrevista preguntas sobre la edad a la que iniciaron la relación con el agresor y la edad de las entrevistadas cuando tuvo lugar el primer episodio de violencia. Igualmente se consideró la duración de la relación y del maltrato.

Escala de Tácticas de Resolución de Conflictos: La entrevista diseñada incluyó ítems de la adaptación de Larraín y 
Bascuñan (1994) de la Escala de Tácticas de Resolución de Conflictos de Straus (1979), para medir la frecuencia de violencia física y psicológica padecida por las entrevistadas. En esta investigación, los ítems utilizados se adaptaron a la realidad nicaragüense. La tipología de respuesta, en escala Likert, comprendía 0 (nunca), 1 (una vez), 2 (a veces), 3 (frecuentemente) y 4 (muy frecuentemente). El análisis KMO (Kaiser-Meyer-Olkin) y la prueba de esfericidad de Bartlett muestran una adecuación muestral aceptable (KMO = .800; Bartlett = 686.42, p = .000). Utilizando como método de extracción Máxima Verosimilitud, los ítems se distribuyeron en dos factores que, en su conjunto, acumulaban el 52.64\% de la varianza.

La matriz factorial rotada convergió en 3 iteraciones (método de rotación Varimax con normalización Kaiser). El Alfa de Cronbach del primer factor es de $\alpha=.822$ y el del segundo factor es de $\alpha=.803$. El Alfa de Cronbach total de la escala indica un nivel aceptable de consistencia interna $(\alpha=$.849). En la Tabla 2 se muestra la matriz de componentes rotada y las saturaciones de los ítems.

Tabla 2

Matriz factorial rotada

\begin{tabular}{lcc}
\hline & Factor 1 & Factor 2 \\
\hline Le ha insultado & .983 & \\
Le ha gritado & .891 & \\
Le ha descalificado frente a otros & .558 & \\
Le ha empujado o sacudido & .544 & \\
Le ha lanzado un objeto & & .771 \\
Rompe objetos intencionalmente & & .769 \\
Ha amenazado con golpearla & & .544 \\
Mantiene silencios prolongados & & .474 \\
Le ha prohibido salir & & .466 \\
Le ha pegado cachetadas & & \\
\hline
\end{tabular}

\section{Análisis de datos}

La elaboración de la base de datos y el procesamiento se realizaron mediante el sistema de análisis estadístico y de gestión de datos SPSS (versión 25.0 para Windows). Para analizar las características sociodemográficas, se utilizaron los siguientes descriptivos: frecuencia, porcentaje, media y desviación típica. Los porcentajes respecto a la interposición de denuncias se obtuvieron tras agrupar las variables de acuerdo a dos categorías: denuncia (valor 1) y no denuncia/retira la denuncia (valor 0 ). Se establecieron ambos grupos a partir de la consideración de que retirar la denuncia (retractación) es considerado un grave problema en Nicaragua (Vázquez et al., 2018). En este sentido, la no interposición de la denuncia y la retirada impiden el inicio de procesos legales respecto al maltrato padecido. En la Escala de Tácticas de Resolución de Conflictos se aplicó la prueba $T$ de Student para muestras independientes (cuando las variables de agrupación estaban compuestas por dos valores), considerando que el resultado estadísticamente significativo tuviese una probabilidad de cometer un error tipo I de $\mathrm{p} \leq$.05. Se utilizó la correlación de Pearson para analizar las relaciones existentes en la Escala de Tácticas de Resolución de Conflictos, así como esta escala y las circunstancias de la violencia padecida. Finalmente, se realizó un análisis de regresión logística binaria con el objetivo de predecir las variables que influyen tanto en la no interposición o retirada de la denuncia.

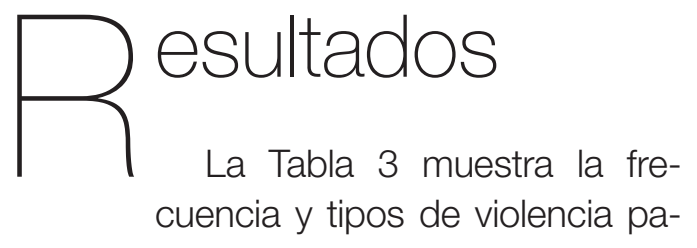

decida por las entrevistadas. Próximos 
al valor frecuentemente se encuentran empujones y sacudidas. En menor memanifestaciones de violencia como gritos, dida los agresores mantuvieron silencios descalificaciones, amenazas con golpes, prolongados hacia sus parejas.

Tabla 3

Agresiones y tipo de violencia padecida por las víctimas de violencia de género de León (Nicaragua).

\begin{tabular}{|c|c|c|c|}
\hline & $\mathrm{n}$ & $\%$ & $\mathrm{M}(\mathrm{DT})$ \\
\hline Ítem 1. Le ha gritado & & & $3.13(.977)$ \\
\hline Nunca & 4 & 2.9 & \\
\hline Una vez & 1 & .7 & \\
\hline A veces & 30 & 22.1 & \\
\hline Frecuentemente & 40 & 29.4 & \\
\hline Muy frecuentemente & 61 & 44.9 & \\
\hline Ítem 2. Le ha insultado & & & $3.19(.939)$ \\
\hline Nunca & 3 & 2.2 & \\
\hline Una vez & 1 & .7 & \\
\hline A veces & 28 & 20.6 & \\
\hline Frecuentemente & 39 & 28.7 & \\
\hline Muy frecuentemente & 65 & 47.8 & \\
\hline Ítem 3. Le ha descalificado frente a otros & & & $2.71(1.327)$ \\
\hline Nunca & 18 & 13.2 & \\
\hline Una vez & 2 & 1.5 & \\
\hline A veces & 30 & 22.1 & \\
\hline Frecuentemente & 37 & 27.2 & \\
\hline Muy frecuentemente & 49 & 36.0 & \\
\hline Ítem 4. Mantiene silencios prolongados & & & $1.67(1.415)$ \\
\hline Nunca & 48 & 35.3 & \\
\hline Una vez & 5 & 3.7 & \\
\hline A veces & 43 & 31.6 & \\
\hline Frecuentemente & 24 & 17.6 & \\
\hline Muy frecuentemente & 16 & 11.8 & \\
\hline Ítem 5. Le ha prohibido salir & & & $2.43(1.391)$ \\
\hline Nunca & 25 & 18.4 & \\
\hline Una vez & 0 & .0 & \\
\hline A veces & 42 & 30.9 & \\
\hline Frecuentemente & 30 & 22.1 & \\
\hline Muy frecuentemente & 39 & 28.7 & \\
\hline Ítem 6. Le ha amenazado con golpearla & & & $2.72(1.281)$ \\
\hline
\end{tabular}




\begin{tabular}{|c|c|c|c|}
\hline & $\mathrm{n}$ & $\%$ & M (DT) \\
\hline Nunca & 16 & 1.8 & \\
\hline Una vez & 2 & 1.5 & \\
\hline A veces & 33 & 24.3 & \\
\hline Frecuentemente & 38 & 27.9 & \\
\hline Muy frecuentemente & 47 & 34.6 & \\
\hline Ítem 7. Rompe objetos intencionalmente & & & $1.65(1.551)$ \\
\hline Nunca & 57 & 39.7 & \\
\hline Una vez & 8 & 5.9 & \\
\hline A veces & 29 & 21.3 & \\
\hline Frecuentemente & 21 & 15.4 & \\
\hline Muy frecuentemente & 24 & 17.6 & \\
\hline Ítem 8. Le ha lanzado objetos & & & $1.76(1.560)$ \\
\hline Nunca & 48 & 35.3 & \\
\hline Una vez & 14 & 10.3 & \\
\hline A veces & 22 & 16.2 & \\
\hline Frecuentemente & 26 & 19.1 & \\
\hline Muy frecuentemente & 26 & 19.1 & \\
\hline Ítem 9. Le ha empujado o sacudido & & & $2.88(1.098)$ \\
\hline Nunca & 8 & 5.9 & \\
\hline Una vez & 3 & 2.2 & \\
\hline A veces & 34 & 25.0 & \\
\hline Frecuentemente & 44 & 32.4 & \\
\hline Muy frecuentemente & 47 & 34.6 & \\
\hline Ítem 10. Le ha abofeteado & & & $1.57(1.479)$ \\
\hline Nunca & 43 & 31.6 & \\
\hline Una vez & 36 & 26.5 & \\
\hline A veces & 19 & 14.0 & \\
\hline Frecuentemente & 13 & 9.6 & \\
\hline Muy frecuentemente & 25 & 18.4 & \\
\hline
\end{tabular}

Como se desprende de la Tabla 4, Como se evidencia en los resultados, a existe una correlación significativa entre mayor frecuencia de violencia física, malos diferentes tipos de agresión, lo que yor es la frecuencia de violencia psicológiquiere decir que estos están relacionados. ca y viceversa. 
Tabla 4

Correlaciones entre manifestaciones de violencia.

\begin{tabular}{|c|c|c|c|c|c|c|c|c|c|}
\hline & İtem 2 & Ítem 3 & Ítem 4 & Ítem 5 & Ítem 6 & Ítem 7 & Ítem 8 & Ítem 9 & Ítem 10 \\
\hline Ítem 1 & $.886^{\star *}$ & $.485^{\star *}$ & -.093 & $.287^{\star \star}$ & $.437^{* *}$ & $.298^{\star \star}$ & $.340^{* \star}$ & $.519^{\star \star}$ & $.289^{\star \star}$ \\
\hline Ítem 2 & 1 & $.579^{* *}$ & -.025 & $.362^{* \star}$ & $.525^{\star \star}$ & $.351^{* *}$ & $.370^{\star \star}$ & $.576^{* *}$ & $.375^{* \star}$ \\
\hline Ítem 3 & - & 1 & .131 & $.227^{\star \star}$ & $.519^{\star \star}$ & $.297^{\star \star}$ & $.303^{\star \star}$ & $.377^{\star *}$ & $.238^{* *}$ \\
\hline Ítem 4 & - & - & 1 & $.309^{* *}$ & .124 & $.353^{* *}$ & $.304^{\star \star}$ & .097 & $.288^{* *}$ \\
\hline Item 5 & - & - & - & 1 & $.458^{\star \star}$ & $.385^{\star \star}$ & $.391^{\star \star}$ & $.302^{\star \star}$ & $.515^{\star \star}$ \\
\hline Ítem 6 & - & - & - & & 1 & $.518^{\star *}$ & $.545^{* \star}$ & $.565^{\star \star}$ & $.366^{* *}$ \\
\hline Item 7 & - & - & - & & & 1 & $.716^{\star \star}$ & $.361^{\star *}$ & $.370^{\star \star}$ \\
\hline Ítem 8 & - & - & - & - & - & - & 1 & $.398^{\star *}$ & $.402^{* \star}$ \\
\hline Ítem 9 & - & - & - & - & - & - & - & 1 & $.468^{* *}$ \\
\hline
\end{tabular}

Nota: ${ }^{*} p \leq 0.05 ;{ }^{* *} p \leq 0.01 ;{ }^{* *} p \leq 0.001$.

En la Tabla 5 se observa la relación entre las diferentes formas de violencia sufrida y la interposición de denuncias. En general, se aprecia mayor frecuencia en cada uno de los actos de violencia entre el grupo de entrevistadas que denunciaron. No obstante, las diferencias estadísticamente significativas entre las entrevistadas que denunciaron respecto a quienes no, se encuentran tanto en la frecuencia con la que sufrieron violencia física (abofetear y amenazar con golpear), psicológica (insultar y prohibir salir de casa) y ambiental (romper objetos intencionalmente). En cuanto a las circunstancias de la violencia en función de la interposición de denuncias, quienes denunciaron comenzaron a padecer violencia a mayor edad ( $M_{\text {denuncian }}=24.72$ vs. $M_{\text {no denuncian }}=21.65$ ). Por otra parte, una mayor frecuencia de la violencia se relaciona con la interposición de denuncias $\left(M_{\text {no denuncian }}=2.66\right.$ (DT $\left.=.836\right)$ vs. $M_{\text {denuncian }}$ $=3.46(\mathrm{DT}=.776) ; \mathrm{t}=-4.678, \mathrm{p}=.000)$.

Tabla 5

Relación entre la interposición de denuncias y las manifestaciones y circunstancias de violencia sufrida

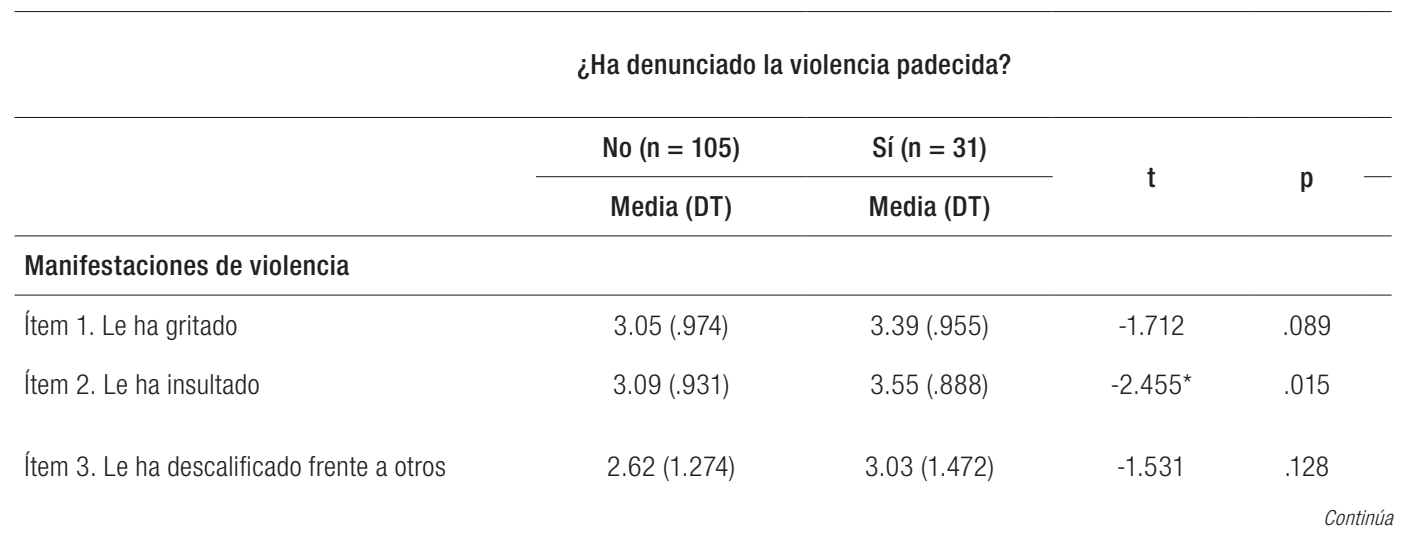




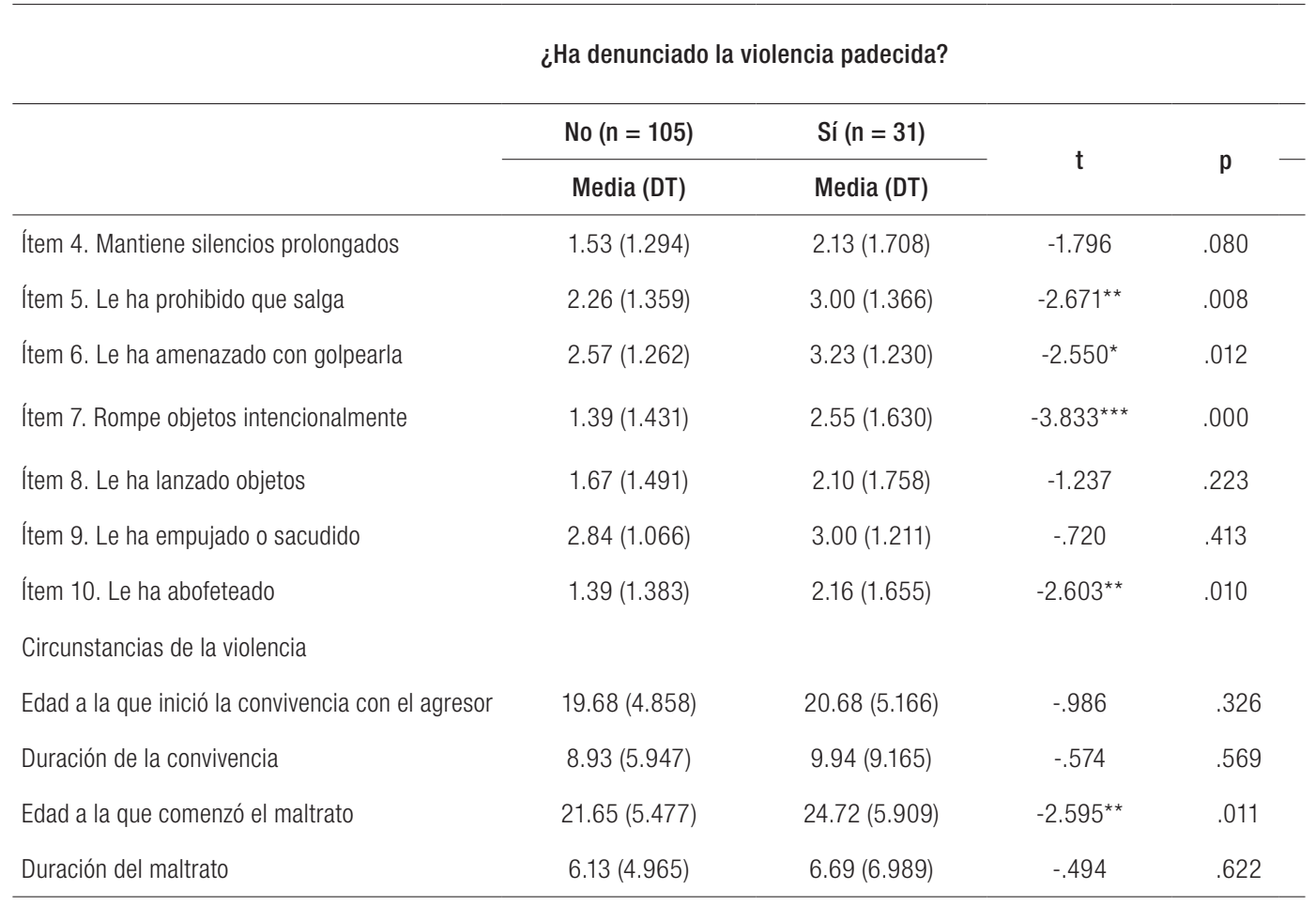

Nota: ${ }^{*} p \leq 0.05 ;{ }^{* \star} p \leq 0.01 ;{ }^{* * *} p \leq 0.001$

Con el objetivo de comprobar qué variables predicen con mayor exactitud la interposición de denuncias se realizó un análisis de regresión logística binaria (véase Tabla 6). En el análisis se incluyeron las variables que resultaron estadísticamente significativas respecto a la denuncia. El modelo que predice que las mujeres denunciaran el maltrato permite una estimación correcta del $86.7 \%$ del total de los casos (el 97.8\% para quienes no cursaron denuncia y el $50 \%$ para quienes sí la tramitaron). La prueba ómnibus proporciona un valor de $\chi^{2}=31.533$ ( $\left.p=.000\right), \mathrm{R}^{2}$ de Nagelkerke estima un valor de ajuste de .349 y la prueba de Hosmer y Lesmeshow $\left(\chi^{2}=18.741 ; p=.016\right)$ indican diferencias significativas en las variables observadas y predichas, lo que lleva a aceptar el modelo resultante.

Tabla 6

Análisis de regresión logística binaria para la predicción de la interposición de denuncias

\begin{tabular}{lcccccc}
\hline & $\beta$ & ET & Wald & $p$ & Exp ( $\beta)$ & IC 95\% \\
\hline Le ha prohibido salir & .393 & .203 & 3.754 & .050 & 1.481 & {$[.995-2.204]$} \\
Edad a la que se comenzaron esas conductas & .113 & .045 & 6.428 & .011 & 1.120 & {$[1.026-1.222]$} \\
Frecuencia del maltrato & & & & & & \\
Constante & 1.304 & .399 & 10.695 & .001 & 3.685 & {$[1.686-8.053]$} \\
\hline
\end{tabular}

Nota: B: coeficiente; ET: error típico; p: probabilidad; $\operatorname{Exp(B):~coeficiente~exponenciado;~IC:~intervalo~de~confianza~al~} 95 \%$. 


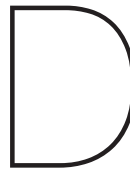

\section{iscusión}

El presente estudio aborda la violencia contra las mujeres como problema de salud pública en un país con bajos niveles de desarrollo, donde la violencia ejercida contra estas se encuentra muy instaurado en las dinámicas familiares como consecuencia de estructuras patriarcales (Bolaños et al., 2009; De Miguel, 2005; Ferrer, 2017; Leonardsson \& San Sebastián, 2017; Medrano, 2012; Rivas et al., 2018). Además, reporta información sobre la prevalencia y tipología de violencia padecida por una muestra de mujeres nicaragüenses que viven en situación de extrema pobreza.

Las entrevistadas llevaban conviviendo con el agresor en torno a los nueve años y el maltrato lo padecieron durante una media de seis años. Como indican trabajos previos, la situación de violencia podría haber conducido a las víctimas de la muestra a una situación de ambivalencia entre el maltrato y el arrepentimiento que las mantendría en la relación (Cala, 2012; Echeburúa et al., 2002; Labrador et al., 2010). Además, en línea con lo reportado en otras investigaciones (Domínguez et al., 2008; Fontanil et al., 2002; Vázquez et al., 2018), todas las participantes padecieron distintos tipos de violencia psicológica y física, aunque la violencia psicológica se produjo con mayor frecuencia. En cuanto a la frecuencia general del maltrato, una de cada cuatro lo padecía diariamente y, en torno a la mitad, lo padecía varias veces a la semana. Otro indicador de la problemática se encuentra en la victimización tanto de violencia psicológica como de violencia física. Entre las manifestaciones de violencia psicológica, sufrieron principalmente gritos, insultos, descalificaciones y amenazas. También se encuentran una frecuencia elevada respecto a padecer empujones/sacudidas por parte de la pareja en el $67 \%$ de los casos. A estos datos hay que añadir la significatividad en las correlaciones entre estas conductas, ya que se observan altos índices de relación entre padecer gritos e insultos, así como las agresiones con objetos. Además, asociadas a las agresiones físicas (empujones y golpes en la cara), se ha encontrado un mayor número de correlaciones con otras manifestaciones de maltrato. Esto en un indicador respecto a que la violencia de género aumenta en frecuencia e intensidad (Labrador et al., 2010; Walker, 2012).

A pesar de la frecuencia del maltrato, más del $75 \%$ de las entrevistadas no interpusieron denuncia o la retiraron, porcentajes muy superiores a los encontrados en otros trabajos (Bolaños et al., 2009; OMS/ OPS, 2012). Cabe señalar entre las mujeres del presente estudio que una mayor frecuencia del maltrato se relaciona con la interposición de denuncias por la violencia padecida. No obstante, se hallaron diferencias estadísticamente significativas entre el grupo de mujeres que interponen denuncia y quienes no en cuanto a la frecuencia con la que se producían insultos, prohibiciones respecto a la libertad de actuar, amenazas, intimidación, así como sufrir violencia física. Los resultados parecen indicar que la violencia ejercida por la pareja o expareja pudo haberse normalizado por las participantes hasta que esta se instauró de forma frecuente en la relación. Los datos aportan información relevante respecto a la percepción de las propias víctimas y la de su entorno, ya que a pesar de que la violencia contra 
las mujeres en la pareja ha dejado de ser un problema del ámbito privado (Bosch et al., 2005), la tolerancia social hacia esta (De Miguel, 2005; Ferrer, 2017; Galtung, 1990) podría estar señalando a la víctima como responsable de su situación al no denunciar el maltrato padecido o retirar la denuncia.

Por otra parte, los resultados muestran que ni el tiempo de convivencia con el agresor ni la duración del maltrato parecen haber mediado en la interposición de denuncias por la violencia sufrida. El análisis de regresión para la predicción de la denuncia indica que entre las variables que en conjunto ayudan a predecir la interposición de denuncias está la limitación respecto a la libertad a actuar (prohibirlas que salgan), la edad de la víctima cuando comenzaron las conductas de maltrato, así como la frecuencia con la que este tenía lugar. Teniendo en cuenta que la ruptura con la relación de maltrato es un momento crucial para las mujeres (Blay, 2013; García-Moreno et al., 2005; Leonardsson \& San Sebastián, 2017; Sagot \& Carcedo, 2000), se tendría que proporcionar una mayor protección en dicho proceso. En el presente estudio, este aspecto se evidencia en que la interposición de denuncias se ha producido cuando las víctimas estaban en mayor riesgo por la frecuencia de las agresiones.

Tenemos que señalar en el presente trabajo una serie de limitaciones. Aunque algunos autores han constatado la validez de la Escala de Tácticas de Resolución de Conflictos en el contexto de Latinoamérica (Paixao, Reichenheim, Moraes, Coutinho \& Veras, 2007), y constituye un referente en los estudios sobre violencia contra las mujeres (Montes-Berges, 2007), ha sido criticada la idoneidad de la Escala al omitir variables relevantes respecto a los antecedentes de violencia, las consecuencias de estos en las víctimas o la tendencia de los hombres a minimizar la responsabilidad de sus actos y de la mujer a atribuirse la culpa (Ferrer \& Bosch, 2005). Además, habría que considerar otras variables que podrían estar afectando a la interposición de denuncias e indagar en factores concomitantes que podrían tener un mayor efecto y repercusión, como el miedo al agresor, tener menores a su cargo y la prevalencia de dependencia económica y emocional en la víctima. Además, dado que la interposición de denuncias es un proceso crucial en la ruptura con la relación de maltrato, habrían de realizarse investigaciones desde perspectivas longitudinales y transculturales. Aún con todo, los resultados ofrecen información sobre la interposición de denuncias en un país sobre el que no abundan estudios (Vázquez et al., 2018).

En conclusión, se hace necesario seguir profundizando en los indicadores que favorecen la interposición de denuncias para que la violencia de género deje de pertenecer al ámbito privado, así como dotar a las víctimas de mecanismos de apoyo dada la dificultad del proceso y los obstáculos a los que se enfrentan a partir de dicho momento, especialmente cuando las mujeres viven en contextos de pobreza (McNaughton et al., 2012; Salazar et al., 2012). No obstante, la falta de dispositivos de ayuda y protección en un país en vías de desarrollo como Nicaragua hacen que la violencia de género siga instaurada y normalizada en las relaciones de pareja. 


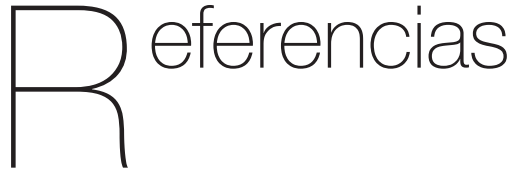

Blay, E. (2013). "Voy o no voy": el recurso a la policía en el caso de la violencia de género. Perspectivas de las víctimas. Estudios Penales y Criminológicos, 33, 369-400.

Bolaños, M.L., Gutiérrez Rodríguez, L., Pérez, E.M., Fanjul, C., Pérez, M.A. \& Barres, O. (2009). Diagnóstico de violencia intrafamiliar y sexual en Nicaragua. Managua: Programa de las Naciones Unidas para el Desarrollo (PNUD).

Bosch, E., Ferrer, V., Alzamora, A. \& Navarro, C. (2005). Itinerarios hacia la libertad: la recuperación integral de las víctimas de violencia de género. Psicología y Salud, 15(1), 1405-1109.

Cala, M.J. (2012). Recuperando el control de nuestras vidas. Reconstrucción de identidades y empoderamiento en mujeres víctimas de violencia de género. Madrid: Instituto de la Mujer.

De Miguel, A. (2005). La construcción de un marco feminista de interpretación: la violencia de género. Cuadernos de Trabajo Social, 18, 231-248.

Domínguez, J.M., García, P. \& Cuberos, I. (2008). Violencia contra las mujeres en el ámbito doméstico: consecuencias sobre la salud psicosocial. Anales de Psicología, 24, 115 - 120. Recuperado de http://revistas. um.es/analesps/article/view/31881

Echeburúa, E., Amor, P.J. \& Corral, P. (2002). Mujeres maltratadas en convivencia prolongada con el agresor: variables relevantes. Acción Psicológica, 2, 135-150.

Doi: https://doi.org/10.5944/ap.1.2.548

Encuesta Nacional de demografía y Salud (2010). Salud y Justicia para las mujeres ante la violencia sexual. Naciones Unidas. Recuperado de http://profamilia.org.co/ docs/ENDS\%202010.pdf

Escobar, A., Quinteros, A., Sánchez Gamonal, S. \& Tardón, B. (2011). Violencia de género: prevención, detección y atención. Madrid: Grupo 5.

Eyre, L.J., Carneiro, R., Remigio, G.A., Soares, A.P., Leite, M.A. \& Magalhaes, R., (2013). Factors associated to the imposition of types of violence against women informed in sentry services. Revista Latino-Americana de Enfermagem, 21(4), 920-927. Doi: http://dx.doi.org/10.1590/ S0104-11692013000400013

Ferrer, V. (2017). Feminismo y Psicología Social. Madrid: Grupo 5.

Ferrer, V. \& Bosch, E. (2005). Introduciendo la perspectiva de género en la investigación psicológica sobre violencia de género. Anales de Psicología, 21(10), 1-10.

Fontanil, Y., Médez-Valdivia, M., Cuesta, M., López, C., Rodríguez, F.J., Herrero, F.J. \& Ezama, E. (2002). Mujeres maltratadas por sus parejas masculinas. Psicothema, 14, 130-138.

Galtung, J. (1990). Cultural Violence. Journal of Peace Research, 27(3), 291-305.

García-Moreno, C., Jansen, H.A.F.M., Ellsberg, M., Heise, L. \& Watts, C. (2005). Estudio multinacional de la OMS sobre salud de la mujer y violencia doméstica contra la mujer. Ginebra: OMS. 
Instituto Nacional de Información y Desarrollo (INIDE, 2015). Anuario Estadístico 2011. Recuperado de http://www.inide.gob.ni/

Labrador, F.J., Fernández-Velasco, M.R. \& Rincón, P. (2010). Características psicopatológicas de mujeres víctimas de violencia de pareja. Psicothema, 22(1), 99105. Doi: http://dx.doi.org/72712699016

Larraín, S. \& Bascuñan, D. (1994). Violencia puertas adentro: La mujer golpeada. Santiago de Chile: Edición Universitaria.

Leonardsson, M. \& San Sebastián, M. (2017). Prevalence and predictors of help-seeking for women exposed to spousal violence in India - a cross-sectional study. BMC Womens Health, 17, 1-15. Doi: http://dx.doi. org/10.1186/s12905-017-0453-4

McNaughton, H.L., Billings, D.L., ParedesGaitan, Y. \& Padilla-Zuniga, K (2012). An assessment of health sector guidelines and services for treatment of sexual violence in El Salvador, Guatemala, Honduras and Nicaragua (2012). Reproductive Health Matters, 20(40), 83-93. Doi: http://dx.doi. org/10.1016/S0968-8080(12)40656-5

Medrano, A. (2012). Desigualdades de género. Bilbao: Mensajero.

Miramontes, M.M. \& Mañas, I. (2018). Vinculación afectiva al agresor en la mujer joven víctima de violencia de género tras la separación. Revista de Psicología, 27(1), 1-12. http://dx.doi. org/10.5354/0719-0581.2018.50741

Montes-Berges, B. (2008). Tácticas para la resolución de conflictos y celos románticos en relaciones íntimas: adaptación y análisis de las escalas CTS2 y CR. Estudios de
Psicología, 29(2), 221-234. Doi: https://doi. org/10.1174/021093908784485138

Organización Mundial de laSalud/Organización Panamericana de Salud (OMS/OPS, 2012). Violencia contra las mujeres en América Latina y El Caribe: análisis comparativo de datos poblacionales de 12 países. Washington, DC: OMS/OPS.

Paixao, C.M., Reichenheim, M.E., Moraes, C.L., Coutinho, E.S. \& Veras, R.P. (2007). Cross-cultural adaptation to Brazil of the instrument Caregiver Abuse Screen (CASE) for detection of abuse of the elderly by caregivers. Cuadernos de Saude Pública, 23(9), 2013-2022.

Rivas, E., Panadero, S., Bonilla, E., Vásquez, R. \& Vázquez, J.J. (2018). Influencia del apoyo social en el mantenimiento de la convivencia con el agresor en víctimas de violencia de género de León (Nicaragua). Informes Psicológicos, 18(1), 145-165. Doi: http://dx.doi.org/10.18566/infpsic.v18n1a08

Sagot, M. \& Carcedo, A. (2000). La Ruta Crítica de las mujeres afectadas por la violencia intrafamiliar en América Latina. Programa Mujer, Salud y Desarrollo. OPS/OMS Pan American Health Organization.

Salazar, M., Högberg, U., Valladares, E. \& Ohman, A. (2012). The supportive process for ending intimate partner violence after pregnancy: the experience of Nicaraguan women. Violence against Women, 18(11), 1257-1278. Doi: http://dx.doi. org/10.1177/1077801212470549

Saltijeral, M.T., Ramos, L. \& Caballero, M.A. (1998). Las mujeres que han sido víctimas de maltrato conyugal: tipos de violencia experimentada y algunos efectos en la salud mental. Salud Mental, 21, 10-18. 
Shorey, R., Brasfield, H., Zucosky, H., Febres, J. \& Stuart, G., (2015). The relation between alcohol use and psychological, physical, and sexual dating violence perpetration among male college students. Violence Against Women, 21(2), 151-164. Doi: http:// dx.doi.org/10.1177/1077801214564689

Sleath, E. \& Smith, L.L. (2017). Understanding the factors that predict victim retractation in police reported allegations of intimate partner violence. Psychology of Violence, 7, 140-149. Doi: http://dx.doi.org/10.1037/ vio0000035

Souza, M., Santos, M.F., Veloso, O., Vieira, M.A., Souza, L.P. \& Cangussu, D. (2014), Reports of violence against women in different life cycles. Revista Latino-Americana de Enfermagem, 22(1), 85-92. Doi: http:// dx.doi.org/10.1590/0104-1169.3186.2388
Straus, M.A. (1979). Measuring intrafamily conflicts and violence: The Conflict Tactics (CT) Scale. Journal of Marriage and the Family, 41, 75-88.

Vázquez, J.J., Rivas, E., Suárez, A.C. \& Panadero, P. (2018). Retraction of complaints among female victims of intimate partner violence living in poverty in Nicaragua. International Journal of Offender Therapy and Comparative Criminology, 62(10), 3151-3167. Doi: http:// dx.doi.org/10.1177/0306624X17725728

Walker, L. (2012). El síndrome de la mujer maltratada. España: Descleé De Brouwer. 